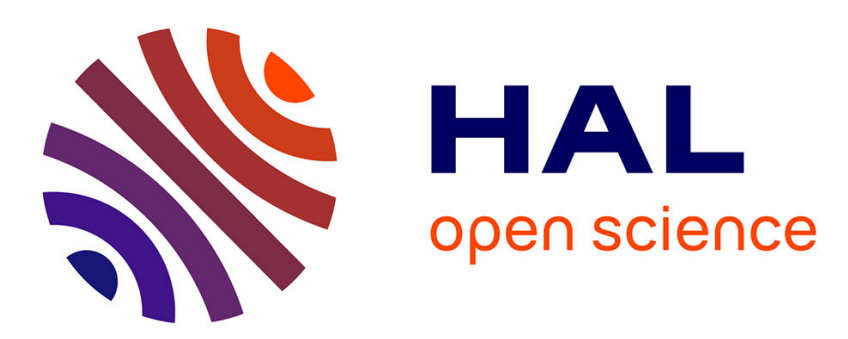

\title{
Supervision of an industrial plant subject to a maximal duration constraint
}

\author{
Abdourrahmane Atto, Claude Martinez, Saïd Amari
}

\section{To cite this version:}

Abdourrahmane Atto, Claude Martinez, Saïd Amari. Supervision of an industrial plant subject to a maximal duration constraint. 9th International Workshop on Discrete Event Systems, May 2008, Göteborg, Sweden. pp.254-259. hal-00362877

\section{HAL Id: hal-00362877 https://hal.science/hal-00362877}

Submitted on 19 Feb 2009

HAL is a multi-disciplinary open access archive for the deposit and dissemination of scientific research documents, whether they are published or not. The documents may come from teaching and research institutions in France or abroad, or from public or private research centers.
L'archive ouverte pluridisciplinaire HAL, est destinée au dépôt et à la diffusion de documents scientifiques de niveau recherche, publiés ou non, émanant des établissements d'enseignement et de recherche français ou étrangers, des laboratoires publics ou privés. 


\section{Supervision of an industrial plant subject to a maximal duration constraint}

\author{
Abdourrahmane M. ATTO
}

\author{
Claude MARTINEZ
}

Saïd AMARI

\begin{abstract}
This paper presents a method for the supervision of an industrial plant. This supervision is aimed at guaranteeing the respect of a maximal duration constraint for some specific processing, and is addressed by considering a discrete event system model for this industrial plant. In this associated model, the time constraint is reduced to elementary constraints whose contributions are taken into account in the state equation of the system, yielding a constrained state equation for the plant. Supervisors are then synthesized by looking for solutions of this constrained state equation.
\end{abstract}

Keywords: Discrete event systems, $(\max ,+)$ algebra, time constraints, supervisor, control.

\section{INTRODUCTION}

This work concerns the supervision of a manufacturing unit that produces rubber hoses for the automotive industry. The development of this industry requires optimization of its productivity, while respecting a strict temporal constraint for specific processing. The sizing of this industrial plant has been solved and validated via computational simulations in [1], and the resource optimization for the manufacturing unit has been treated in [2]. The problem addressed in this paper concerns the supervision of the plant in order to guarantee the respect of a strict temporal constraint for the thermal treatments involved. The supervision is aimed at guaranteeing that this time constraint is met without impacting significantly the production rate of the manufacturing unit. It is shown that this supervision can be performed thanks to analytical techniques.

The industrial plant studied can be modelled as a Discrete Event System (DES). Several approaches have been proposed for the analysis of DES these last few decades [3]. A DES can be modelled with a Timed Event Graph (TEG) [4], [5] when it represents phenomena requiring synchronisations and excluding competition as well as conflict. The analysis of such a system can then be described with linear equations in $(\max ,+)$-algebra [4], [6]. The industrial plant under consideration satisfies these assumptions. Thus, in order to guarantee the time constraint imposed, we propose solutions based on the constrained $(\max ,+)$ state equation of the TEG model of the plant.

Performance evaluation is of great interest in the literature on the $(\max ,+)$-algebra topic [4], [6], [7]. In this application, the performance of a supervisor will be measured according to the maximum production throughput of the supervised plant. According to this particular performance

EMIG Niamey, am.atto@ieee.org

IUT Nantes, Claude.Martinez@univ-nantes.fr

ENS Cachan, said.amari@lurpa.ens-cachan.fr measure, we can classify supervisors between those which slow down the production throughput and those which preserve this production rate. The cycle time of such a plant modelled as a TEG corresponds to the eigenvalue of the matrix associated with its graph [6], [8], [9], the production throughput is the inverse of the cycle time. Similar problems of meeting time constraints have been recently addressed with different approaches[10], [11], [12], [13].

This work is organised as follows. Section II briefly recalls the fundamentals of $(\max ,+)$ algebra, section III presents a TEG model for the plant and gives its corresponding linear $(\max ,+)$ model. Section IV addresses the supervision problem and provides a simple way for synthesizing supervisors for time constrained systems. This section provides 3 supervisors for the manufacturing plant and classifies them by showing that some preserve the production throughput of the plant, in comparison to that of the non-supervised plant. Finally, section $\mathrm{V}$ gives a conclusion and addresses perspectives to extend this work.

\section{II. (max, +$)$ ALGEBRA}

This section briefly recalls the fundamentals of $(\max ,+)$ algebra, which is largely used for the analysis of DES. Further details on this theory may be found in [4], [6], [14], [15]. In what follows, $\mathcal{D}$ denotes a set.

Definition 1 (Monoid): A monoid is an algebraic set with an associative internal operation and an identity element.

Definition 2 (Semiring): $(\mathcal{D}, \oplus, \otimes)$ is a semiring if:

- $(\mathcal{D}, \oplus)$ is a commutative monoid. Its identity element is denoted by $\epsilon$ (null element).

- $(\mathcal{D}, \otimes)$ is a monoid. Its identity element is denoted by $e$ (unit element).

- Multiplication $\otimes$ distributes over addition and every $x \in$ $\mathcal{D}$ is such that $x \otimes \epsilon=\epsilon \otimes x=\epsilon$.

Definition 3 (Dioid): A dioid $(\mathcal{D}, \oplus, \otimes)$ is an idempotent semiring (every $x \in \mathcal{D}$ is such that $x \oplus x=x$ ).

Hereafter, the product $a \otimes b$ will be denoted $a . b$ or $a b$ when there is no possible confusion.

Example 1: Examples of dioids:

- Let $\mathbb{R}$ be the set of real numbers. $(\mathbb{R} \cup\{-\infty\}, \max ,+)$ is a commutative dioid for which $\epsilon=-\infty$ and $e=0$. This dioid is denoted by $\mathbb{R}_{\max }$ and is called $(\max ,+)$ algebra.

- Let $(\mathcal{D}, \oplus, \otimes)$ be a dioid and $\mathcal{D}^{n \times n}$ the set of square matrices of order $n$ over $\mathcal{D}$. $\left(\mathcal{D}^{n \times n}, \oplus, \otimes\right)$ is a dioid called a matrix dioid. The sum and the matrix product are defined as follows: if $A=\left(A_{i j}\right), B=\left(B_{i j}\right)$, then $(A \oplus B)_{i j}=A_{i j} \oplus B_{i j}$ and $(A \otimes B)_{i j}=\bigoplus_{k=1}^{n} A_{i k} \otimes$ 


\section{$B_{k j}$. The null element of the matrix dioid is the matrix composed of $\epsilon$. The unit matrix is the matrix with $e$ on the main diagonal and $\epsilon$ elsewhere.}

\section{TEG REPRESENTATION AND LINEAR $(\max ,+)$ MODEL FOR A MANUFACTURING UNIT}

A Petri net consists of places, directed arcs, and transitions. Directed arcs connect places and transitions (there is no direct connection between two places or between two transitions). TEGs are a subclass of Petri Nets in which every place is connected to only one input and one output transitions. According to the nature of the problem tackled in this paper, we focus on the particular case where crossing transitions is instantaneous. In such cases, temporisations are set only over places [5]. The temporisation associated with each place corresponds to the minimum duration of a specific process running in this place and marked by a token. Each transition $x_{j}$ is associated with a function that gives the firing time for the $k^{\text {th }}$ occurrence $x_{j}(k)$.

A nice example of TEG is that of the manufacturing unit of the industrial plant under consideration. This unit specialises in manufacturing rubber tubes for automotive equipment and is represented in figure 1. This figure represents three con-

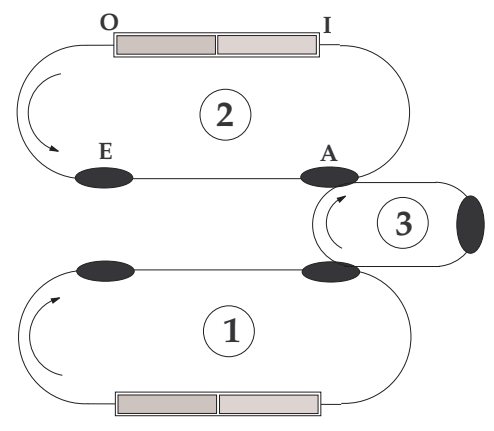

Fig. 1. Manufacturing unit.

veyor belts connected in loops. Loops $\mathbf{1}$ and $\mathbf{2}$ are identical. Each one is composed of a loading station (A, on loop 2) where parts subject to heat treatment are fixed on specific pallets, a unloading station (E) where parts are dismounted, and a furnace (IO cells). The furnace itself consists of two parts, a heating zone and a cooling zone. The parts are subjected to high temperatures during the time they spent in the first half of the furnace. Then, they are cooled in the second half. After cooling of parts, pallets are brought to the unloading station where an operator removes the parts from the pallets and dispatches them in batches towards another unit of the production workshop. The transport device is not always available for the evacuation of treated parts and this could cause an accumulation of pallets at the unloading station. In such cases, saturation may occur at the entry of the unloading station, causing the system to block. The pallets present in the furnace then exceed their processing time and the embarked products are burned and lost. Thus, for this application, the time spent in the heating zone is critical: the maximal heating time should not be exceeded even when non-evacuation of treated products occurs at the unloading station.

Loops $\mathbf{1}$ and $\mathbf{2}$ being identical, we can restrict our attention in one loop ( $\mathbf{2}$ in the sequel). We adopt an analytical approach to solve the supervision problem for this application. This manufacturing unit reveals synchronisations between loops. Indeed, loading pallet is possible only if an empty pallet and parts to supply it are present at the loading station $\mathbf{A}$. In the same way, availability of the transport device is necessary at the unloading station to take away the treated parts (station E) and make available an empty pallet for forthcoming use. This type of industrial plant requiring synchronisations can be modelled as a TEG. The TEG model of this application is that of figure 2. In this graph, transitions are associated

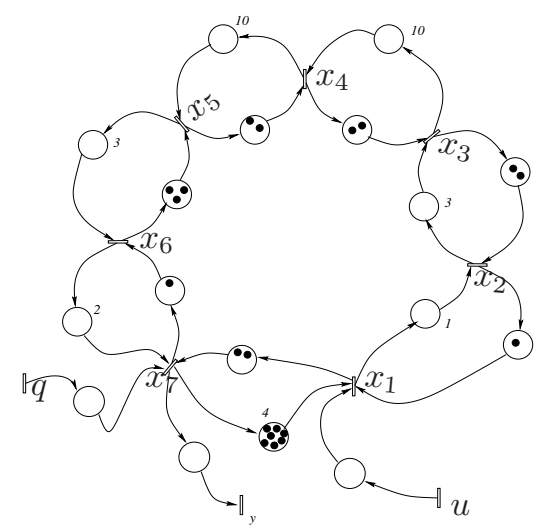

Fig. 2. TEG model for loop $\mathbf{2}$ of the industrial plant.

with the following events:

- $u$ : arrival of the parts;

- $x_{1}$ : beginning of the loading operation;

- $x_{2}$ : starting transport to the furnace;

- $x_{3}$ : entry to the heating zone of the furnace;

- $x_{4}$ : entry to the cooling zone of the furnace;

- $x_{5}$ : starting transport to the evacuation zone;

- $x_{6}$ : beginning of the unloading operation;

- $x_{7}$ : part evacuation;

- $q$ : transport device (may be present or absent).

- $y$ : departure of the parts.

The input transition $u$ models the arrival of parts to be treated and the transition $q$ models the transport device for evacuating finished parts. When the transport device fails, saturation can occur because of non-evacuation of treated products. The output transition $y$ corresponds to actually treated and evacuated parts. Crossing transition $x_{i}$ corresponds to the occurrence of an event, for example, crossing $x_{1}$ corresponds to the beginning of the loading operation on a pallet, $x_{2}$ to the end of this operation and the beginning of transport to the furnace. Operation durations are indicated close to places; for example, the transfer of a pallet from the loading station to the entry of the furnace (station $\mathbf{I}$ ) is about 3 time units.

Tokens (in places) model the resources of the manufacturing unit: pallets, operators, capacity of conveyors, etc. For instance, the transfer time from unloading station $\mathbf{E}$ 
to loading station $\mathbf{A}$ is four time units. In addition, there are actually seven free pallets and there remain two places available on the conveyor (in the graph of figure 2).

The state vector, $x$, of this TEG is composed of transitions $x_{1}, x_{2}, \cdots, x_{7}$; and the input vector, $v$, is composed of transitions $u$ and $q$. The state and output equations that describe the dynamic behaviour of the TEG of figure 2 are given in $(\max ,+)$-algebra by

$$
\begin{aligned}
& x_{1}(k+1)=x_{2}(k) \quad \oplus 4 x_{7}(k-6) \oplus u(k+1), \\
& x_{2}(k+1)=1 \quad x_{1}(k+1) \oplus x_{3}(k-1), \\
& x_{3}(k+1)=3 \quad x_{2}(k+1) \oplus x_{4}(k-1), \\
& x_{4}(k+1)=10 x_{3}(k+1) \oplus x_{5}(k-1), \\
& x_{5}(k+1)=10 x_{4}(k+1) \oplus x_{6}(k-2), \\
& x_{6}(k+1)=3 x_{5}(k+1) \oplus x_{7}(k), \\
& x_{7}(k+1)=2 x_{6}(k+1) \oplus x_{1}(k-1) \oplus q(k+1), \\
& y(k)=x_{7}(k) .
\end{aligned}
$$

In these equations, $\oplus$ denotes the max operator and the multiplication corresponds to the natural element addition in the set of real numbers (see [4], [6] for further details about $(\max ,+)$-algebra). These equations yield a matrix representation where state $x(k)$ at time $k$ depends on states $x(k), x(k-1), x(k-2), x(k-3), x(k-7)$ and on input $v(k)$. However, there exists a simplified representation of the TEG state of the form: $x(k+1)=A_{0} x(k+1) \oplus A x(k) \oplus B v(k+1)$. Indeed, a place with $m$ tokens and temporisation $\alpha$ is equivalent to $m$ places, each of them having only one token and temporisation $\alpha_{i}$, with $\sum \alpha_{i}=\alpha$. According to this decomposition, the TEG model of the manufacturing unit is that of figure 3 (reduction from depth 7 to depth 1 ).

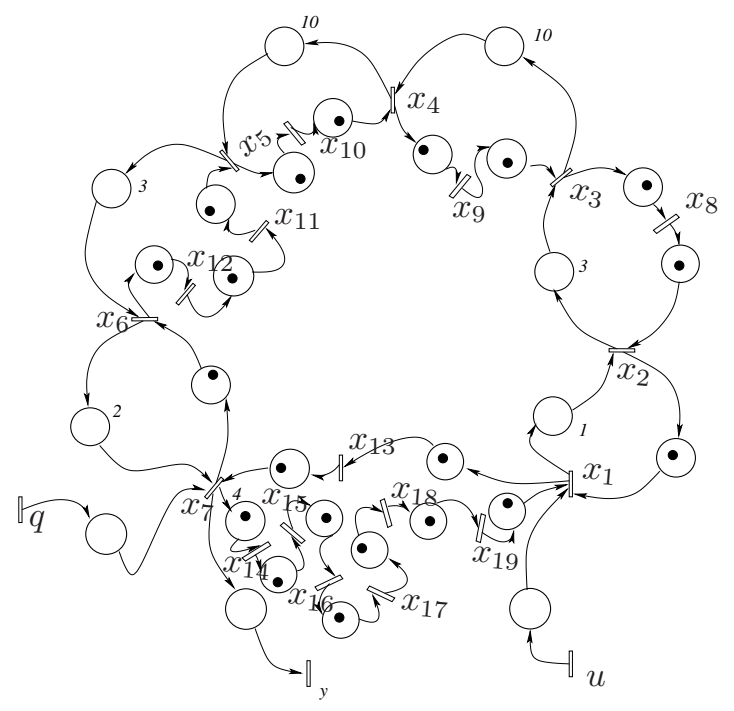

Fig. 3. Simplified model for loop 2 of the manufacturing unit.

The dynamic behaviour of the simplified TEG obtained (figure 3 ) is described by a system of the form:

$$
\left\{\begin{array}{l}
x(k+1)=H_{0} x(k+1) \oplus H_{1} x(k) \oplus K_{0} v(k+1), \\
y(k)=S x(k),
\end{array}\right.
$$

where matrices $K_{0}, H_{0}, H_{1}$ and $S$ are omitted here because of their large size and the limited length of the present paper.
After reduction (using the Kleene star operator, see [6], [4]), system Eq. (2) is equivalent to:

$$
\left\{\begin{array}{l}
x(k+1)=H x(k) \oplus K v(k+1), \\
y(k)=S x(k),
\end{array}\right.
$$

where $H$ and $K$ are given below. In these matrices, $e=$ 0 denotes the unit element; and the null element, $-\infty$, is replaced by a dot.

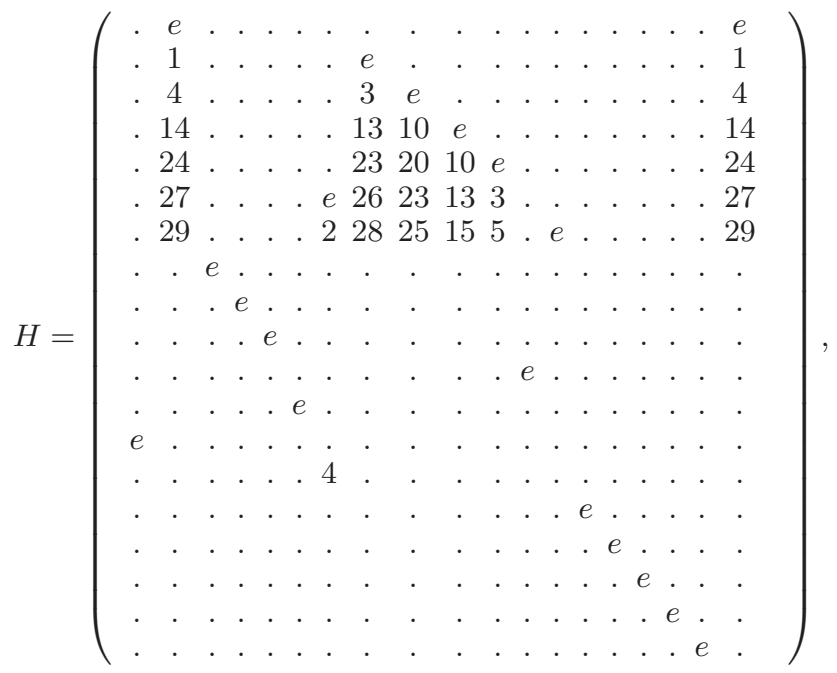

and

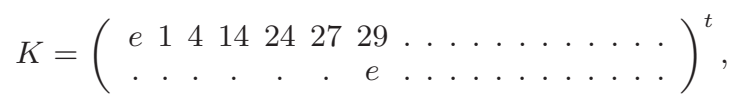

where $t$ denotes matrix transposition.

\section{SUPERVISION OF THE INDUSTRIAL PLANT}

\section{A. Maximal duration constraints}

The minimum duration of tokens in places is expressed by temporisations of these places. On the other hand, if we wish to express a maximum duration in a place, we must then add an additional constraint. Consider the TEG represented in figure 4.

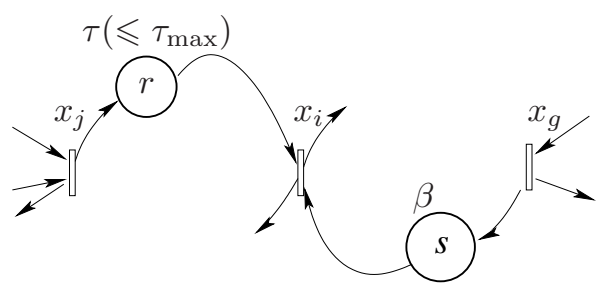

Fig. 4. Temporal constraint

Let $p_{i j}$ be the place linking transition $t_{j}$ to transition $t_{i}$, and $p_{i g}$ the place linking transition $t_{g}$ to transition $t_{i}$. The marking (number of tokens) of place $p_{i j}$ is $r$. If we want to enforce a maximum time duration $\tau_{\max }$ to tokens in place $p_{i j}$, then the following inequality must be satisfied:

$$
x_{i}(k) \leqslant \tau_{\max } x_{j}(k-r) .
$$

In addition, according to the graph of figure 4, transition $t_{i}$ firing is governed by:

$$
x_{i}(k)=\tau x_{j}(k-r) \oplus \beta x_{g}(k-s),
$$


where $s$ is the number of tokens in place $p_{i g}, \tau \leqslant \tau_{\max }$ is the temporisation of place $p_{i j}$, and $\beta$ is the temporisation of place $p_{i g}$.

From Eqs. (4) and (5) we derive the (necessary and sufficient) condition under which a token will not exceed the duration constraint $\tau_{\max }$ in place $p_{i j}$ :

$$
\beta x_{g}(k-s) \leqslant \tau_{\max } x_{j}(k-r) .
$$

Note that for TEGs, a place with $m>1$ tokens can be decomposed in $m$ places with one token. For this reason, we focus on the special case where $s-r=1$. The maximum duration constraint Eq. (4) is then expressed in the following form:

$$
\beta x_{g}(k-1) \leqslant \tau_{\max } x_{j}(k) .
$$

The next example shows how to express the maximum duration constraint in the form of Eq. (7).

Example 2: Consider the TEG given in figure 5. Durations $\tau_{1}^{\max }$ and $\tau_{2}^{\max }$ are the normal durations of tokens

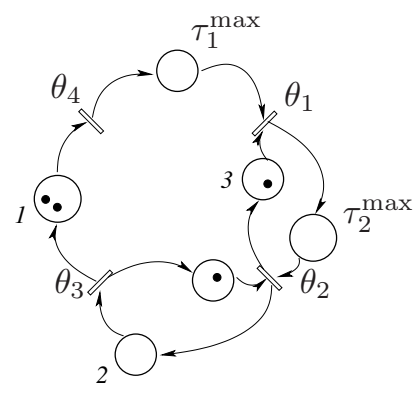

Fig. 5. TEG with two duration constraints.

in corresponding places and these durations should not be exceeded. The constraints are:

$$
\begin{aligned}
& \text { (a) } \theta_{1}(k) \leqslant \tau_{1}^{\max } \theta_{4}(k), \\
& \text { and } \\
& \text { (b) } \theta_{2}(k) \leqslant \tau_{2}^{\max } \theta_{1}(k) .
\end{aligned}
$$

These constraints are respected if:

$$
\begin{aligned}
& \text { (a) true if } 3 \theta_{2}(k-1) \leqslant \tau_{1}^{\max } \theta_{4}(k) \text {, } \\
& \text { (b) true if } \theta_{3}(k-1) \leqslant \tau_{2}^{\max } \theta_{1}(k),
\end{aligned}
$$

that is, if

$$
\left(\begin{array}{cccc}
\epsilon & \epsilon & e & \epsilon \\
\epsilon & 3 & \epsilon & \epsilon
\end{array}\right) \theta(k) \leqslant\left(\begin{array}{cccc}
\tau_{2}^{\max } & \epsilon & \epsilon & \epsilon \\
\epsilon & \epsilon & \epsilon & \tau_{1}^{\max }
\end{array}\right) \theta(k+1),
$$

with $\theta=\left(\theta_{1} \theta_{2} \theta_{3} \theta_{4}\right)^{t}$.

Consider a TEG governed by a state equation of the form (general state representation)

$$
\left\{\begin{array}{l}
x(k+1)=A x(k) \oplus B v(k+1), \\
y(k)=C x(k)
\end{array}\right.
$$

Assume that we want to impose on this TEG, a set of time constraints of the form

$$
E x(k) \leqslant F x(k+1) .
$$

where $E$ and $F$ are $\ell \times n$ matrices, $\ell$ being the number of constraints and $n$ the length of the state vector $x$.
Supervisors guaranteeing that the constraints Eq. (9) are met can be calculated by applying a state modification (constrained state equation) given by

$$
x(k+1)=(A \oplus M) x(k) \oplus B v(k+1),
$$

where $M$ (supervision matrix) is a matrix satisfying

$$
E \leqslant F M \text {. }
$$

\section{B. Constraint expression for the industrial plant}

The supervision is aimed at preventing parts being lost because of possible failure in the transport device. The $(\max ,+)$-equation that governs the time spent by a part in the heating zone of the furnace is derived from the dynamic behaviour of the simplified TEG of figure 3 and is:

$$
x_{4}(k+1)=10 x_{3}(k+1) \oplus x_{10}(k) .
$$

To avoid losing parts, a product should not exceed 10 time units in the heating zone of the furnace (place that links transition $x_{3}$ to transition $x_{4}$ in figures 2 and 3). Thus, the constraint will be respected by forcing

$$
x_{4}(k+1)=10 x_{3}(k+1),
$$

Taking Eq. (12) into account, condition Eq. (13) will be satisfied iff:

$$
x_{10}(k) \leqslant 10 x_{3}(k+1) .
$$

Denoting

$$
\begin{aligned}
& Q_{1}=(\ldots \ldots \ldots \text {. . . . . . }) \text {, } \\
& Q_{2}=(\ldots 10 \ldots \ldots \ldots \ldots \ldots)
\end{aligned}
$$

the constraint condition Eq. (14) is thus of the form Eq. (9): $Q_{1} x(k) \leqslant Q_{2} x(k+1)$.

\section{Supervision of the manufacturing unit}

The dynamic behaviour of the manufacturing unit is described with the $(\max ,+)$ system Eq. (3). The maximum duration constraint imposes $Q_{1} x(k) \leqslant Q_{2} x(k+1)$. A supervisor guaranteeing respect of the duration constraint can be calculated by searching for a matrix $M_{0}$ (supervision matrix) satisfying

$$
Q_{1} \leqslant Q_{2} M_{0}
$$

The only non null element of $Q_{1}$ being $\left(Q_{1}\right)_{1,10}=e$, it is sufficient to consider the solutions of:

$$
e \leqslant \bigoplus_{j=1}^{19}\left(Q_{2}\right)_{1, j}\left(M_{0}\right)_{j, 10}
$$

that is,

$$
e \leqslant 10\left(M_{0}\right)_{3,10} \text {. }
$$

The smallest positive (least restrictive) solution of the latter equation is $\left(M_{0}\right)_{3,10}=e$. The supervision obtained from this solution involves adding to the graph of figure 3 , a place having a single token (with no temporisation because $\left(M_{0}\right)_{3,10}=e \equiv 0$ ) from transition $x_{10}$ to transition $x_{3}$.

Let ${ }_{m} x_{3}$ be the state of transition $x_{3}$ after supervision. Firing of transition ${ }_{m} x_{3}$ is then subject to

$$
{ }_{m} x_{3}(k+1)=x_{3}(k+1) \oplus x_{10}(k) .
$$


Transition $x_{10}$ being an auxiliary variable derived from the expansion of the original model of the manufacturing unit (represented by the graph of figure 2), we do not have access to this transition in practice: it is neither controllable, nor observable [16]. But from Eq. (3) we derive that $x_{10}(k)=$ $x_{5}(k-1)$, and equation Eq. (18) becomes

$$
{ }_{m} x_{3}(k+1)=x_{3}(k+1) \oplus x_{5}(k-1) .
$$

The resulting graph modification involves adding a single place with two tokens from transition $x_{5}$ to transition $x_{3}$. State modification driven by Eq. (19) leads to the supervisor represented in figure 6 . This supervision involves imposing only two tokens in the circuit $x_{3} \rightarrow x_{5} \rightarrow x_{3}$ which corresponds to the IO cells of the plant (figure 1), that is, the whole furnace (heating and cooling zone). It is easy to check that if we do this, no product will remain more than 10 time units in the heating zone of the furnace because there will be a free place in the cooling zone. This supervision guarantees that parts (a maximum of two parts) entering the furnace cannot be lost even when saturation occurs at the evacuation station.

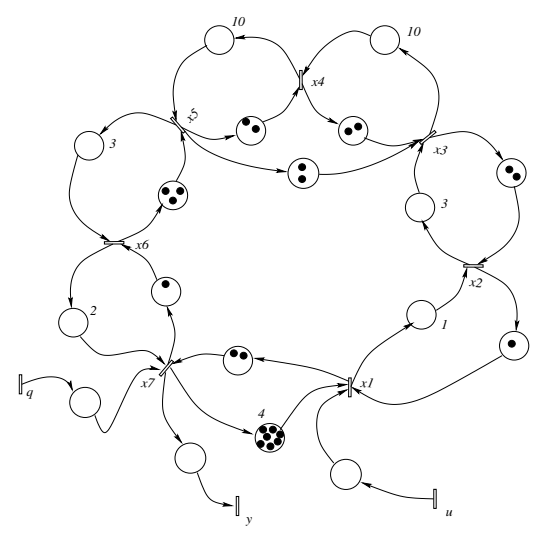

Fig. 6. First supervision.

In a similar way, we can obtain other supervisors from Eq. (19) and by taking into account other equations of Eq. (1) (for commandability reasons). Indeed, from the fifth equation of Eq. (1), we have $x_{5}(k-1)=10 x_{4}(k-1) \oplus x_{6}(k-4)$. In this equation, $10 x_{4}(k-1)$ represents the normal incrementation of the process due to the dynamic behaviour of the TEG, and $x_{6}(k-4)$ represents availability of a resource (conveying to the unloading station). Non-evacuation of a part treated affects the availability of the resource: there is no more place at the unloading station to receive new parts and treated parts accumulate in the conveyor. It follows that $x_{5}(k-1)=$ $x_{6}(k-4)$. From this latter equation and Eq. (19), we thus obtain the state modification:

$$
{ }_{m} x_{3}(k+1)=x_{3}(k+1) \oplus x_{6}(k-4) .
$$

This new supervision involves adding a single place with five tokens from transition $x_{6}$ to transition $x_{3}$. This leads to the supervision presented in figure 7. The supervisor imposes a maximum of five tokens in circuit $x_{3} \rightarrow x_{6} \rightarrow x_{3}$. Thus, imposing five tokens in this circuit makes it possible to guarantee that no parts will remain more than 10 time units in the heating zone: five places are available in the circuit $x_{4} \rightarrow x_{6} \rightarrow x_{4}$.

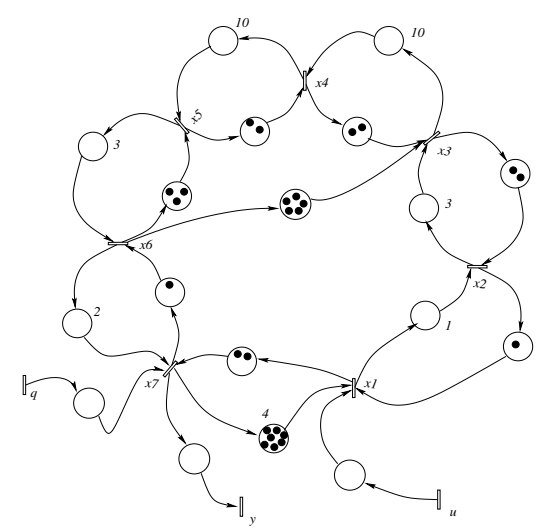

Fig. 7. Second supervision.

Finally, and in a similar way, a third supervisor is calculated from Eq. (20) and the sixth equation of Eq. (1). Indeed, we have $x_{6}(k-4)=3 x_{5}(k-4) \oplus x_{7}(k-5)$, where $3 x_{5}(k-4)$ represents the normal incrementation of a process due to the dynamic behaviour of the TEG and $x_{7}(k-5)$ represents availability of a resource (unloading operator). Non-evacuation of treated parts only affects resource availability and it follows that $x_{6}(k-4)=x_{7}(k-5)$. We thus obtain from this equation and Eq. (20) the corresponding state modification:

$$
{ }_{m} x_{3}(k+1)=x_{3}(k+1) \oplus x_{7}(k-5) .
$$

By proceeding in this way, the supervision involves imposing six tokens in circuit $x_{3} \rightarrow x_{7} \rightarrow x_{3}$. The supervision obtained is given in figure 8 .

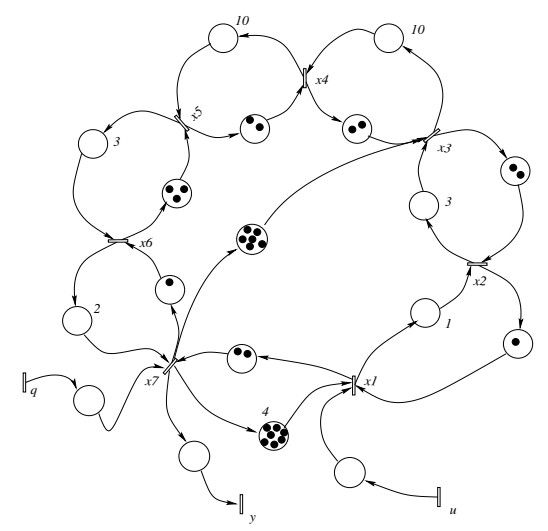

Fig. 8. Third supervision.

\section{Classification of supervisors}

This section discusses the classification of supervisors synthesised in section IV-C. Classification is addressed by comparing the production throughput yielded by systems 
"TEG+supervisor", in comparison to the production throughput of the non-supervised manufacturing unit. For this purpose, we compute cycle times associated with the TEGs of figures 2, 6, 7 and 8. Recall that the cycle time is the inverse of the production throughput.

Let $\lambda$ be the cycle time of the non-supervised manufacturing unit (figure 2). The following procedure (see [8] or [17]) makes it easy to determine $\lambda$ : for every circuit $i$ (sequence of vertices and arcs which allows a direct connection from $i$ to $i$ ) of the graph, determine the ratio

$$
\lambda_{i}=\frac{\text { Sum of cycle temporisations }}{\text { Number of tokens in the cycle }} .
$$

Then, $\lambda$ is the maximum of $\lambda_{i}$.

From the above procedure, the cycle time of the nonsupervised unit is:

$$
\lambda=\max \left\{\frac{33}{7}, \frac{1}{1}, \frac{3}{2}, \frac{10}{2}, \frac{10}{2}, \frac{3}{3}, \frac{2}{1}, \frac{0}{13}\right\}=5,
$$

and the cycle times are $\lambda^{\prime} \max \{\lambda, 20 / 2\}=10, \lambda^{\prime \prime}=$ $\max \{\lambda, 23 / 5\}=5$ and $\lambda^{\prime \prime \prime}=\max \{\lambda, 25 / 6\}=5$ of the unit supervised according to figures 6,7 and 8 respectively.

The cycle time $\lambda^{\prime}$ of the supervised TEG of figure 6 is greater than $\lambda$. Thus, the supervision represented in figure 6 affects the production throughput of the industrial plant. In contrast, $\lambda^{\prime \prime}=\lambda^{\prime \prime \prime}=\lambda$ : supervisors represented in figures 7 and 8 preserve the initial production throughput of the industrial plant. Comparing the TEGs in figures 7 and 8 , the cycle time yielded by adding the supervisor's place, are $23 / 5$ and $25 / 6$, but both supervisors lead to the same resulting throughput for the plant and they are therefore equivalent according to production throughput criteria.

In order to illustrate the plant functioning with and without supervision, assume that the unloading operator may not be present before 100 time units have passed. Figure 9 shows the firing of transitions $u, x_{3}, x_{4}$, and $y$ for the non-supervised plant and ${ }_{m} x_{3}$ replaces $x_{3}$ for the supervised plant, with the third supervisor (figure 8). We observe that the delay between firing $x_{3}(7)$ and $x_{4}(7)$ is 86 for the non-supervised plant: products are lost, while supervision ensures that the time constraint is met, which is 10 time units in the place that links transitions ${ }_{m} x_{3}(k)$ to $x_{4}(k)$.

\section{CONCLUSiOn}

This paper presents a method for the supervision of a manufacturing plant subject to strict time constraints. The method proposed involves injecting the constraint in the state equation of the TEG model associated with the plant and solving the constrained state equation just obtained. This analysis makes it possible to synthesise supervisors aimed at guaranteeing maximum duration constraints. In order to classify supervisors, the use of performance criterion such as the cycle time could be considered. The approach used in this work for a specific TEG can be extended by considering general states and time constraint expressions. This generalisation will be addressed in future work.

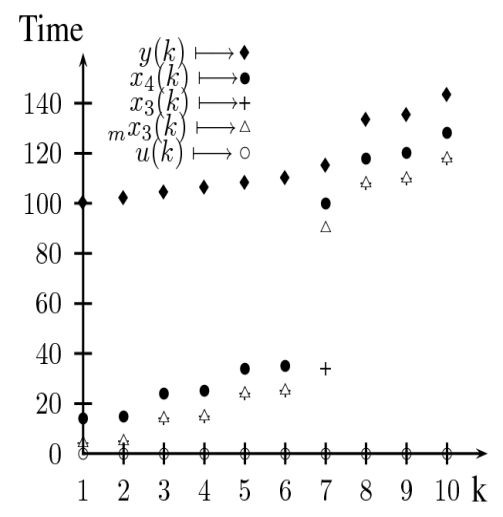

Fig. 9. Behaviour of the plant in both unsupervised and supervised cases. Note that the duration between firings of $x_{3}(7)$ and $x_{4}(7)$ exceeds the time constraints (10 time units) in the case of the non-supervised plant. This problem no longer exists for the supervised plant $\left(m_{m} x_{3}(7)\right)$

\section{REFERENCES}

[1] C. Martinez and P. Castagna, "Sizing of an industrial plant using tight time constraints using complementary approaches: $(\max ,+)$ theory and computer simulation," Elsevier, Simulation Practice and Theory, vol. 11, pp. pp 75-88, 2003.

[2] S. Amari, I. Demongodin, J. Loiseau, and C. Martinez, "Sizing and cycle time of an industrial plant using dioïd algebra," t. M. on Advanced Computer Systems Production System Design Supply Chain Management (ACS'02-SCM), Ed., 2002, pp. pp 99-106.

[3] C. Cassandras and S. Lafortune, Introduction to discrete event systems, K. Academic, Ed. Kluwer Academic, 1992.

[4] S. Gaubert, "Théorie des systémes linéaires dans les dioïdes," Ph.D. dissertation, École des Mines de Paris, 1992.

[5] T. Murata, "Petri nets: Properties, analysis and applications," I. Proceedings, Ed., vol. 77, no. 4, 1989, pp. pp 541-580.

[6] F. Baccelli, G. Cohen, G. Olsder, and J. Quadrat, Synchronization and Linearity, Wiley, Ed. Wiley, 1992.

[7] G. Cohen, P. Moller, J. Quadrat, and M. Viot, "Algebraic tools for the performance evaluation of discrete event systems," I. P. S. issue on Discrete Event Systems, Ed., 1985.

[8] G. Cohen, D. Dubois, J. Quadrat, and M. Viot, "Analyse du comportement périodique des systèmes de production par la théorie des dioïdes," INRIA, Tech. Rep. 191, 1983.

[9] S. Gaubert, "Resource optimization and $(\min ,+)$ spectral theory," IEEE Trans. on Automatic Control, vol. 40, no. 11, pp. pp 1931-1934, 1995.

[10] P. Spacek, M. Manier, and A. Moudni, "Control of an electroplating line in the max and min algebras," International Journal of Systems Science, vol. 30, no. 7, pp. pp 759-778, 1999.

[11] J. Kim and T. Lee, "Schedule stabilization and robust timing control for time-constrained cluster tools," I. I. C. on Robotics and T. Automation, Taipei, Eds., 2003, pp. pp 1039-1044.

[12] S. Amari, J. Loiseau, and I. Demongodin, "Control of linear min-plus systems under temporal constraints," t. I. C. on Decision, Control, and S. the European Control Conference 2005, Seville, Eds., 2005, pp. pp $7738-7743$.

[13] L. Houssin, S. Lahaye, and J. Boimond, "Just in time control of constrained (max, +)-linear systems," Journal of Discrete Event Dynamic Systems, vol. 17, pp. pp 159-178, 2007.

[14] G. Cohen, "Dioids and discrete event systems," in Lect. Notes. in Control and Inf. Sci, P. of the 11th Conf. on Anal. and O. of Systems: Discrete Event Systems, Eds., no. 199. Springer, Sophia Antipolis, 1994.

[15] L. Libeaut, "Sur l'utilisation des diö̈des pour la commande des systèmes à événements discrets," Ph.D. dissertation, ECN - Université de Nantes, 1996.

[16] C. Commault, "Feedback stabilisation of some event graph models," IEEE Trans. on Automatic Control, 1998.

[17] H. Hillion and J. Proth, "Performance evaluation of job-shopsystems using timed event-graphs," IEEE Trans. on Automatic Control, vol. 34, no. 1 , pp. 3-9, 1989. 